Published in final edited form as:

Proc SPIE. 2014 March 12; 8949: . doi:10.1117/12.2041839.

\title{
Fast fluorescence holographic microscopy
}

\author{
Wan Qin ${ }^{1}$, Xiaoqi Yang ${ }^{1}$, Yingying $\mathrm{Li}^{1,2}$, Xiang Peng ${ }^{2}$, Xinghua $\mathbf{Q u}^{3}$, Hai Yao ${ }^{1}$, and Bruce Z. \\ Gao ${ }^{1, *}$ \\ ${ }^{1}$ Department of Bioengineering and COMSET, Clemson University, Clemson, SC 29634, USA \\ ${ }^{2}$ Institute of Optoelectronics, Shenzhen University, Shenzhen, Guangdong 518060, China \\ ${ }^{3}$ State Key Laboratory of Precision Measuring Technology and Instruments, Tianjin University, \\ Tianjin 300072, China
}

\begin{abstract}
FINCHSCOPE is a new technology of fluorescence holographic microscopy. It has been successfully applied to recording high-resolution three-dimensional fluorescence images of biological specimens without the need for scanning. In this study, we revealed and analyzed an intrinsic phenomenon, called ghost lens effect, on spatial light modulator which is the core element enabling the incoherent correlation in the FINCHSCOPE. The ghost lens effect can degrade the imaging quality by introducing multiple spherical waves with different focal lengths into the correlation and thus increasing the noise in the recorded holograms.
\end{abstract}

\section{Keywords}

Fluorescence holography; spatial light modulator; FINCHSCOPE; ghost lens effect

\section{INTRODUCTION}

In recent years, three-dimensional (3D) imaging techniques such as laser scanning and confocal imaging have been applied to fluorescence microscopy [1-4]. In most of these 3Dimaging schemes, the 3D image is captured by scanning the 3D object, either point by point or section by section. The requirement of a sequential scan to collect $3 \mathrm{D}$ information is the major drawback of these methods: It is time-consuming and requires a precise 3Dpositioning device. Scanning may also by increasing exposure.

Fresnel Incoherent Correlation Holography (FINCH) is a recently introduced technique to record 3D information from incoherent sources such as incoherently illuminated objects and fluorescent objects [5-11]. It captures all of the 3D information in one hologram such that the 3D information is encoded in a series of Fresnel zone plates. Generally, in the FINCH system, light is reflected, or emitted, from a 3D object, propagates through a spatial light modulator (SLM), and is recorded by a digital camera. The SLM splits each spherical beam originating from each object point into two beams (object beam and reference beam), a

(C) 2014 SPIE

*Corresponding author's zgao@clemson.edu. 
plane wave and a spherical wave or two spherical waves with different curve radiuses, such that the FINCH system operates as a single channel incoherent interferometer producing holograms of separate object points. Incoherent summation of the self-interferences from every object point creates the Fresnel hologram of the 3D object. Subsequently, the hologram is digitally reconstructed by applying the Fresnel transform [12]. The FINCH was first tested for mercury arc lamp illumination [5] and later for fluorescent objects [6]. The method was then successfully used to design a fluorescence holographic microscope, called FINCHSCOPE [13-15].

FINCHSCOPE is a motionless holographic microscopy system based on FINCH and is used to record high-resolution three-dimensional fluorescence images of biological specimens without the need for scanning. In this study, we reveal a ghost lens effect of SLM that can degrade the imaging quality of FINCHSCOPE. The ghost lens effect stems from the discrete pixel values uploaded to the LCoS screen of the SLM. It is a nature of digital devices and is difficult to eliminate. However, we have found that the level of the ghost lens effect depends on the focal length of the diffractive lens modulated by the SLM.

\section{EXPERIMENTAL METHODS}

\subsection{INCHSCOPE Setup}

The FINCHSCOPE setup is schematically shown in Figure 1, which was mainly composed of an objective, a filter set, a mercury arc lamp, an SLM, and a CCD camera. The sample employed in this study was fluorescent polymer microspheres (Duke Scientific Corp., 35-8, 48um diameter, Excitation Maxima $468 \mathrm{~nm}$, Emission Maxima $508 \mathrm{~nm}$ ). An excitation filter (Thorlabs MDF-FITC excitation filter, 475/35) was used to select excitation wavelengths centered at $475 \mathrm{~nm}$ from a mercury arc lamp. The emitted fluorescence with a wavelength centered at $508 \mathrm{~nm}$ passed from an infinity-corrected microscopic objective (Motic EF-N Plan, 10×, 0.25 NA) through a dichroic mirror (Thorlabs MDF-FITC dichroic filter, 470-490 $\mathrm{nm} / 508-675 \mathrm{~nm}$ ) and an emission filter (Thorlabs MDF-FITC emission filter, 530/43), and was reflected at 12 degree from a phase-only SLM (Holoeye PLUTO NIR2, 1080×1920 pixels) onto a digital cooling EMCCD camera (Andor iXon+, 14 bit, $1024 \times 1024,13 \mu^{2}$ ).

Here we briefly describe the FINCHSCOPE approach as follows (For detail of the working principle of the FINCHSCOPE, please refer to Ref. [14]): A phase map is displayed on the SLM, creating a composition of two different spherical diffractive lenses (one of them having infinite focal length). In this way, a single wavefront originating from each object point is split by the SLM into two mutually coherent wavefronts with two different spherical curves. These two beams propagate in the same direction towards the camera and mutually interfere on the sensor chip. The intensity pattern of the interference originating from the same point source is in the shape of the Fresnel zone plate. The depth location of this radiating point is encoded by the density of Fresnel zone plate rings, and its transverse location is encoded directly by the transverse location of the Fresnel zone plate center. Incoherent accumulation of interference patterns of each object point in the specimen results in a hologram that is recorded by the camera and introduced into a computer. To eliminate the twin image and the bias term resulting from each single hologram, three incoherent 
holograms are required to record sequentially, each with a different phase factor of the SLM pattern.

\subsection{Ghost Lens Effect}

To determine the ghost lens effect of the SLM, we conducted a series of experiments with the FINCHSCOPE setup. The method is illustrated in Figure 2. A polarizer was placed in front of the SLM to achieve an optimum modulation. A sample that contains single-layer fluorescence beads was located at the working distance of the objective, and the fluorescence images of the sample were the main criteria to determine the ghost lens effect in the experiment.

The phase maps loaded on the SLM to generate desired diffractive lenses were created by MATLAB. Here we use $f_{2}$ to denote the equivalent focal length of the diffractive lens, and we will show later that there are several ghost lenses accompanied with the desired diffractive lens. The ghost lenses are derivatives that inevitably exit beside the desired lens on the SLM. Theoretically, determining ghost lenses generated by the SLM would require a fixed distance between the sample and the objective (fixed $f$ in Fig. 2), right at its working distance. Then, by changing the distance between the SLM and the CCD camera, $d$, and recording all the $d$ value where a focused image exists, we can find out how many ghost lenses there are and what focal lengths they have. However, if $d$ is too large, the signal captured by the CCD camera will be very weak, which will decrease the signal to noise ratio, and the light intensity between recorded images would be significantly different. To avoid this situation, an alternative method was used in our experiment: We fixed $d$ at the imaging plane of the desired diffractive lens, and then, by changing the sample-objective distance (the deviation of the sample position away from the working distance is denoted as $\Delta f$ ), we recorded each image that contains focused sample. The black lines in Fig. 2 stand for the optical path of imaging a sample at the working distance of the objective, while the red lines represent the optical path with a certain $\Delta f$. When the sample is not located at the working distance of the objective ( $\Delta f$ is not zero), only if there is another equivalent lens (ghost lens), besides the desired diffractive lens, on the SLM, could the CCD camera record a focused image. This method also guaranteed a relatively stable light intensity captured by the CCD camera.

\section{RESULTS AND DISCUSSIONS}

\subsection{Experimental Results}

The experiment was separated into two groups of tests. In the first test, the focal length of the diffractive lens loaded on the SLM, $f_{d}$, was set to be $400 \mathrm{~mm}$, and accordingly $d$ was also $400 \mathrm{~mm}$. In the second test, $f_{2}$ and $d$ were set to be $600 \mathrm{~mm}$. The fluorescence imaging results of the two tests are shown in Figs. 3 and 4, respectively. During the first test, as depicted in Fig. 3, 6 focused images were obtained at different $\Delta f$ when vertically moving the sample. This fact demonstrated that there were 6 lenses superimposed on the SLM when the $f_{d}=400 \mathrm{~mm}$ diffractive lens was uploaded, 1 desired lens $\left(f_{d}=400 \mathrm{~mm}\right)$ and 5 ghost lenses. Among the 6 fluorescence images, Fig. 3(d) is of the best signal-to-noise ratio, because it was imaged by the $f_{d}=400 \mathrm{~mm}$ lens which was dominant among the others. The 
other lenses were derivatives produced due to the discrete pixel values and limited resolution of the SLM (refer to Fig. 6). Figures 3(a), (b), (c), (e) and (f) were imaged by ghost lenses so that serious noises comparable to signals occurred. The noise in each image was mainly composed of the defocused images of the other 5. Similarly, Fig. 4 presents 5 fluorescence images produced with the desired $f_{d}=600 \mathrm{~mm}$ lens (Fig. 4(d)), and other 4 ghost lenses (Figs. 4(a), (b), (c) and (e)). .

It has been reported that the gaps between SLM pixels can form an equivalence lens. Normally it can be minimized but not completely eliminated by a polarizer. In order to separate the effect of gaps from ghost lenses, another group of tests were taken with a blank screen (equal phases) on the SLM (Fig. 5). As a result, when $\mathrm{d}=400 \mathrm{~mm}$, a focused image (Fig. 5(a)) was obtained at the position of $\Delta f=+0.432 \mathrm{~mm}$, which matches the result of Fig. 3(c). Similarly, as d=600 mm, Fig. 5(b) matches Fig. 4(c).

\subsection{Discussion}

Now let's discuss how ghost lenses are generated. SLM reshapes the incident light by modulating its phase. A phase map loaded on SLM is displayed as a gray-scale image in which each pixel represents a phase value. When uploading a lens onto an SLM, one can control the focal length by changing the density of the Fresnel zone plate. However, the Fresnel zone plate on SLM can never be perfect due to the limited resolution of the SLM screen. This can be clearly seen in Fig. 6, which shows 3 phase maps of Fresnel zone plates to be uploaded onto SLM. Theoretically, a phase map should look like a series of gradually changing, concentric rings. Whereas, in reality, the phase map will not only contain one series of concentric rings but also plenty of others on the sides. The right side of Fig. 6 is the zoom-in view of the phase maps, displaying a series of rings centered at different places on the horizontal midline. The densities of those derivative rings are different, which could function as ghost lenses placed behind/in front of the desired lens.

The reason why the derivative rings occur is that SLM images are composed using pixels, which have discrete values. SLM is not capable of continuously modulating the phase of light, as a real optical lens. This can be simply illustrated with Fig. 7. It is clear that the error rate will increase when fewer pixels are available to represent a continuous value range. In another word, higher density of concentric rings of the Fresnel zone plate (that is, shorter focal length of the SLM-generated diffractive lens) can result in more ghost lenses that contribute more noise to FINCHSCOPE. Nevertheless, the ghost lens effect of SLM has potential to be applied to new imaging techniques other than holography, e.g. multiple-layer imaging in real time.

\section{ACKNOWLEDGEMENT}

This work was supported by National Institute of Health (P20RR021949 and 1k25h1088262-01); National Science Foundation (MRI CBET-0923311 and SC EPSCoR RII EPS-0903795 through SC GEAR program); Guangdong Provincial Department of Science and Technology, China (2011B050400011); and the grant established by the State Key Laboratory of Precision Measuring Technology and Instruments (Tianjin University). 


\section{REFERENCES}

[1]. Schilling BW, Poon TC, Indebetouw G, et al. Three-dimensional holographic fluorescence microscopy. Optics Letters. 1997; 22(19):1506-1508. [PubMed: 18188283]

[2]. Garcia-Sucerquia J, Xu WB, Jericho SK, et al. Digital in-line holographic microscopy. Applied Optics. 2006; 45(5):836-850. [PubMed: 16512525]

[3]. Indebetouw G, Zhong WW. Scanning holographic microscopy of three-dimensional fluorescent specimens. Journal of the Optical Society of America a-Optics Image Science and Vision. 2006; 23(7):1699-1707.

[4]. Johnson SC. Fluorescence holographic microscopy speeds 3-D imaging. Laser Focus World. 2008; 44(5):25-26.

[5]. Rosen J, Brooker G. Digital spatially incoherent Fresnel holography. Optics Letters. 2007; 32(8): 912-914. [PubMed: 17375151]

[6]. Rosen J, Brooker G. Fluorescence incoherent color holography. Optics Express. 2007; 15(5): 2244-2250. [PubMed: 19532459]

[7]. Rosen J, Siegel N, Brooker G. Theoretical and experimental demonstration of resolution beyond the Rayleigh limit by FINCH fluorescence microscopic imaging. Optics Express. 2011; 19(27): 26249-26268. [PubMed: 22274210]

[8]. Katz B, Rosen J, Kelner R, et al. Enhanced resolution and throughput of Fresnel incoherent correlation holography (FINCH) using dual diffractive lenses on a spatial light modulator (SLM). Optics Express. 2012; 20(8):9109-9121. [PubMed: 22513622]

[9]. Kelner R, Rosen J, Brooker G. Enhanced resolution in Fourier incoherent single channel holography (FISCH) with reduced optical path difference. Optics Express. 2013; 21(17):2013120144. [PubMed: 24105559]

[10]. Rosen J, Brooker G. Fresnel incoherent correlation holography (FINCH): A different way of 3D imaging. Laser Focus World. 2013; 49(3):49-51.

[11]. Siegel N, Rosen J, Brooker G. Faithful reconstruction of digital holograms captured by FINCH using a Hamming window function in the Fresnel propagation. Optics Letters. 2013; 38(19): 3922-3925. [PubMed: 24081089]

[12]. Yamaguchi I, Zhang T. Phase-shifting digital holography. Optics Letters. 1997; 22(16):12681270. [PubMed: 18185816]

[13]. Siegel N, Rosen J, Brooker G. Reconstruction of objects above and below the objective focal plane with dimensional fidelity by FINCH fluorescence microscopy. Optics Express. 2012; 20(18):19822-19835. [PubMed: 23037035]

[14]. Rosen J, Brooker G. Non-scanning motionless fluorescence three-dimensional holographic microscopy. Nature Photonics. 2008; 2(3):190-195.

[15]. Brooker G, Siegel N, Rosen J, et al. In-line FINCH super resolution digital holographic fluorescence microscopy using a high efficiency transmission liquid crystal GRIN lens. Optics Letters. 2013; 38(24):5264-5267. [PubMed: 24322233] 


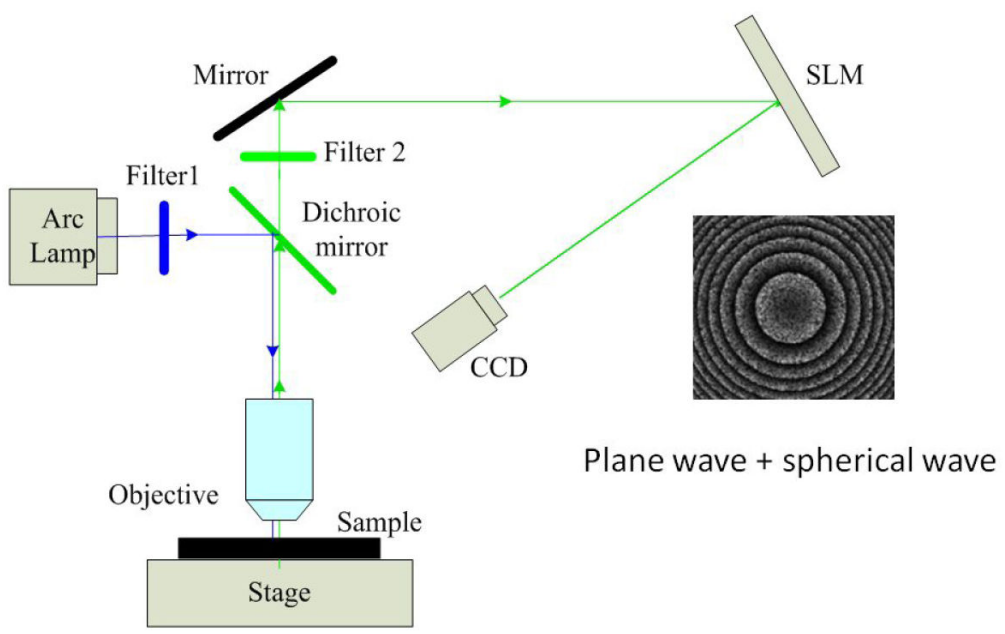

Fig. 1.

Schematic of the FINCHSCOPE. Filter1, Filter2 and Dichroic mirror: Thorlabs MDF-FITC filter set; objective: Motic EF-N Plan, 10×, 0.25 NA; SLM: Holoeye PLUTO NIR2, $1080 \times 1920$ pixels; CCD: Andor iXon+ cooling EMCCD camera, 14 bit, $1024 \times 1024,13$ $\mu \mathrm{m}^{2}$. 


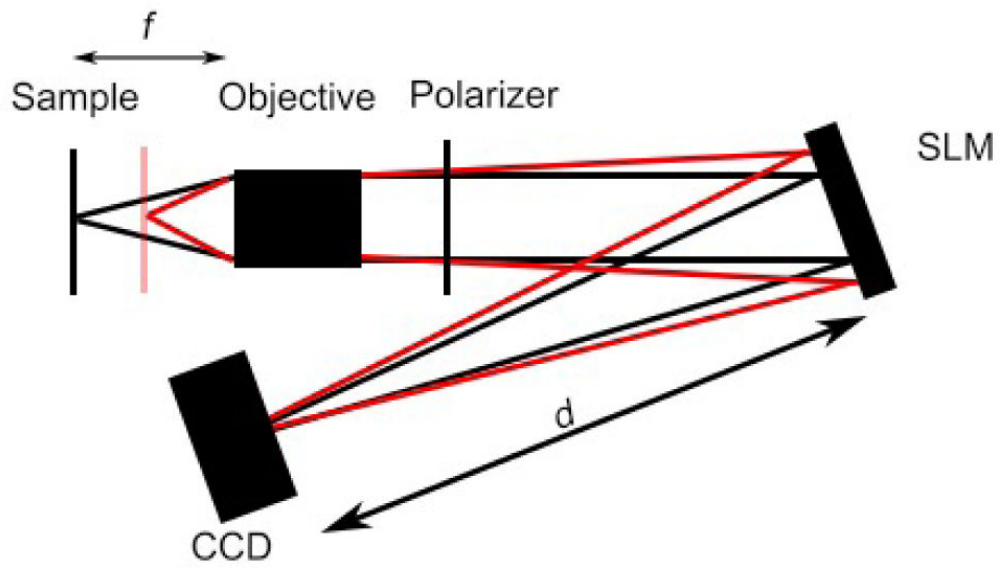

Fig. 2.

Schematic of the method of determining the ghost lens effect. $f$ : working distance of the objective; $d$ : distance between SLM and CCD; the black and red lines represent two sets of optical paths with different sample-objective distances. 


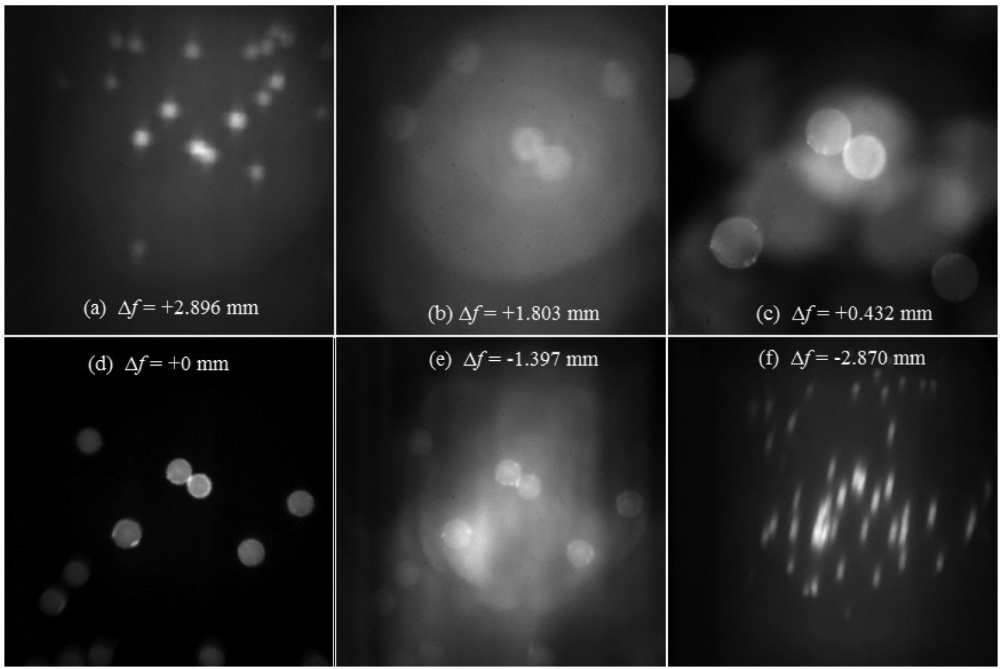

Fig. 3.

Fluorescence images captured with different sample-to-objective distances $\left(f_{d}=400 \mathrm{~mm}\right)$. 


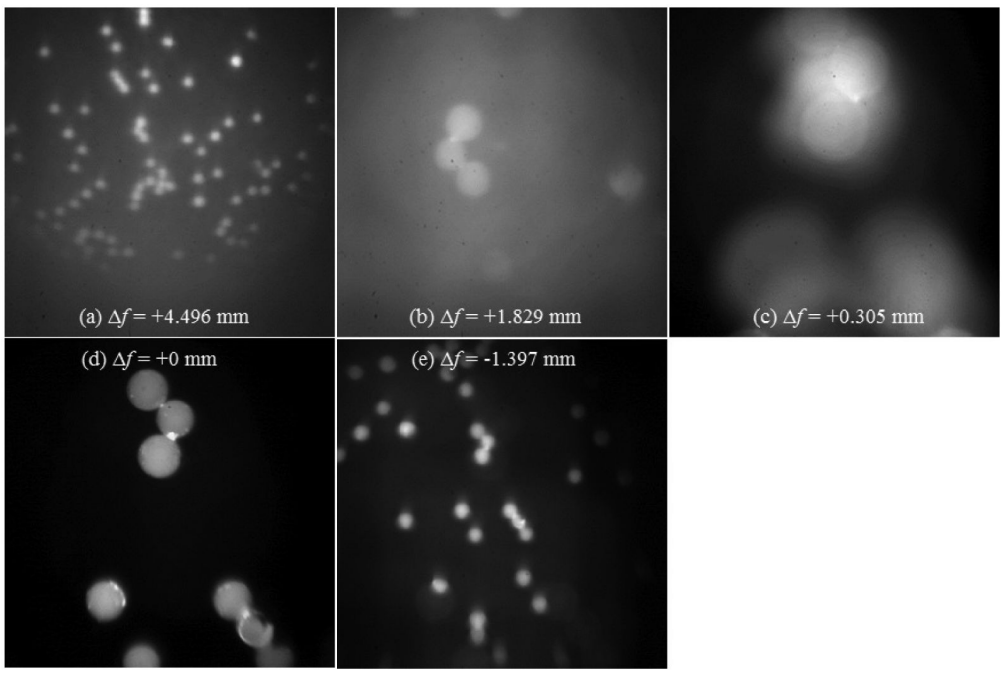

Fig. 4.

Fluorescence images captured with different sample-to-objective distances $\left(f_{d}=600 \mathrm{~mm}\right)$. 


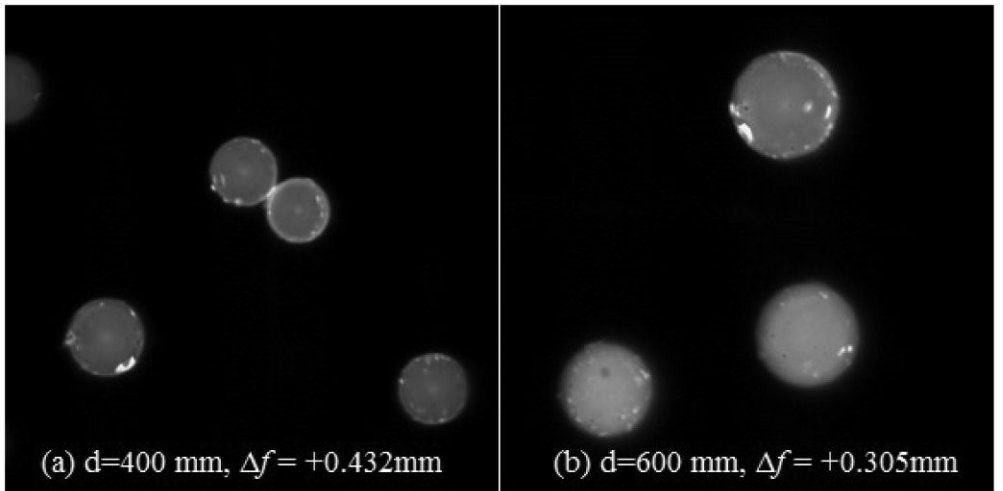

Fig. 5.

Fluorescence images captured with the lenses resulted from pixel gaps of the SLM. 

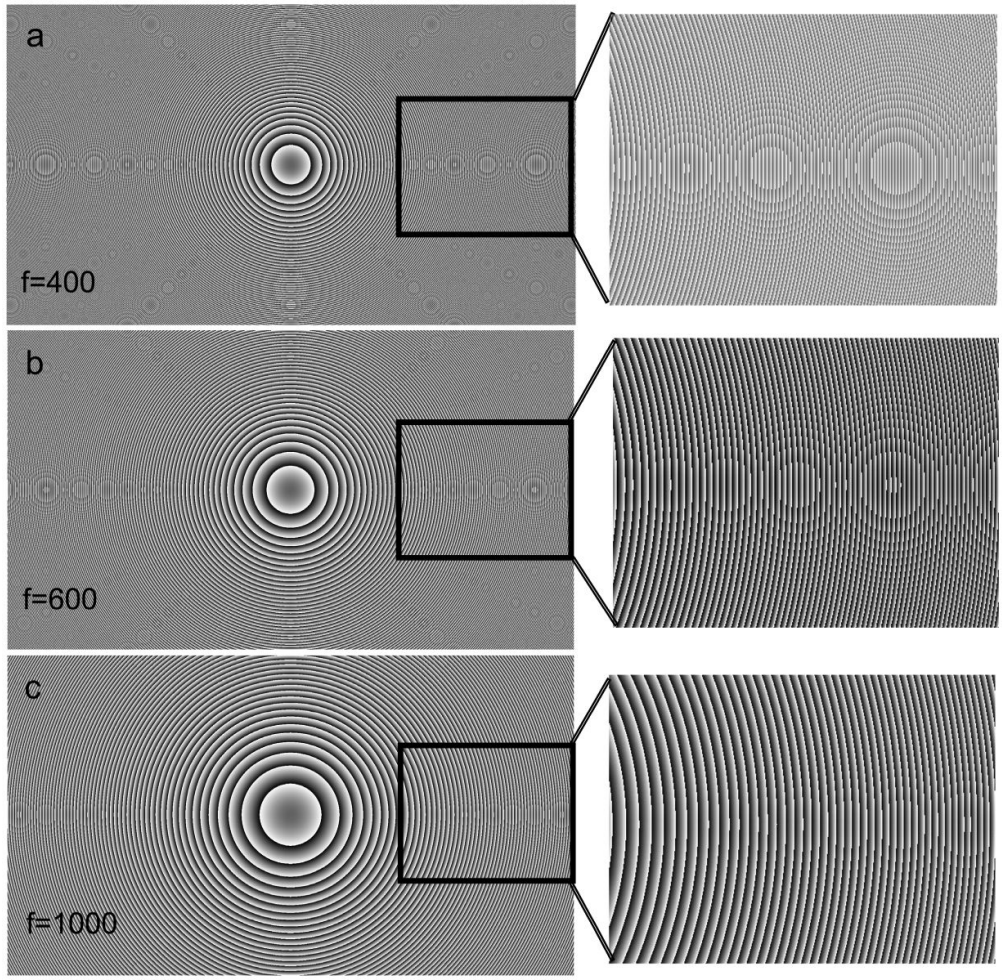

Fig. 6.

Phase maps of Fresnel zone plates with different focal lengths 

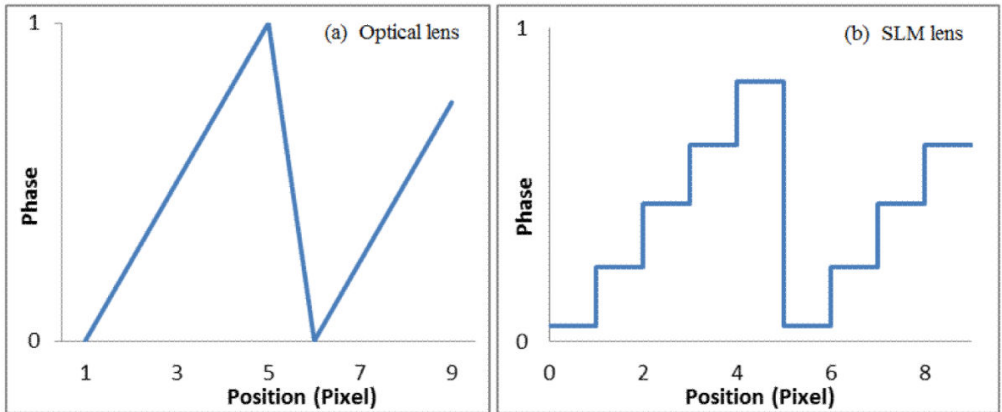

Fig. 7.

Comparison of the capability of phase modulation between an optical lens and an SLMbased lens 